\title{
Effect of magnetic fields on Lithium depletion of solar-type stars
}

\author{
Tanda $\mathbf{L i}^{1}$ and Shaolan $\mathbf{B i}^{1,2}$ \\ ${ }^{1}$ Department of Astronomy, Beijing Normal University, Beijing 100875, China \\ email: litanda@hotmail.com \\ ${ }^{2}$ Key Laboratory of Solar Activity, National Astronomical Observatories, Chinese Academy of \\ Sciences, Beijing 100012, China \\ email: bisl@bnu.edu.cn
}

\begin{abstract}
To study the effect of magnetic fields on lithium depletion and explain the characters of lithium evolution in solar-type stars, stellar models including Tayler-Spruit dynamo-type field are constructed. We compare the theoretical results with the time scales of lithium depletion suggested by Sestito \& Randich (2005) and obtain very good agreements.
\end{abstract}

Keywords. lithium abundances, stellar evolution, magnetic field

\section{Introduction}

Lithium is easily burned at relatively low temperature $\left(\sim 2.5 \times 10^{6} \mathrm{~K}\right)$ in stellar interior, and hence it is an extraordinarily sensitive diagnostic of stellar structure and evolution. A large amount of studies has been devoted to the understanding of the observational feature of $\mathrm{Li}$ and its evolution. Lithium depletion and dispersion has been found in $\mathrm{G}$ and late-F stars of open cluster during both pre-main sequence (PMS) and main sequence (MS). However, with the standard or classical stellar model which includes convection only as a mixing process we could not reproduce similar characters of lithium evolution. To explain the lithium depletion during the MS stage, a number of extra-mixing mechanisms are operating to transport lithium from the outer CZ to deeper regions and lithium depletion.

\section{Overview}

The observed feauters of lithium could be mainly described in two points as follow: (i) lithium depletes from PMS and decrease with age; (ii) dispersion of lithium was found at a fix $T_{\text {eff }}$ in open clusters. Furthermore, Sestito \& Randich (2005) performed a new homogeneous analysis of all the lithium data available in the literature for main sequence stars. More detailed features of lithium depletion was obtained in their work, which is the so-called time scale of lithium evolution. The time scale can be schemed as follows: (i) a small amount of PMS Li destruction; (ii) plateau during the early stages of the MS; (iii) MS depletion; (iv) plateau at old ages.

However, the present models, including rotation-induced mixing (Chaboyer et al. (1995); Deliyannis \& Pinsonneault (1997)) and gravity waves (Montalban \& Schatzmann (2000)) could not reproduce all these charaters, especially the plateau at old ages. Since lithium is an extraordinarily sensitive diagnostic of extra-mixing process in stellar interior, to fitting the observed features we need improve our knowledge about extra-mixing in stellar interior. 


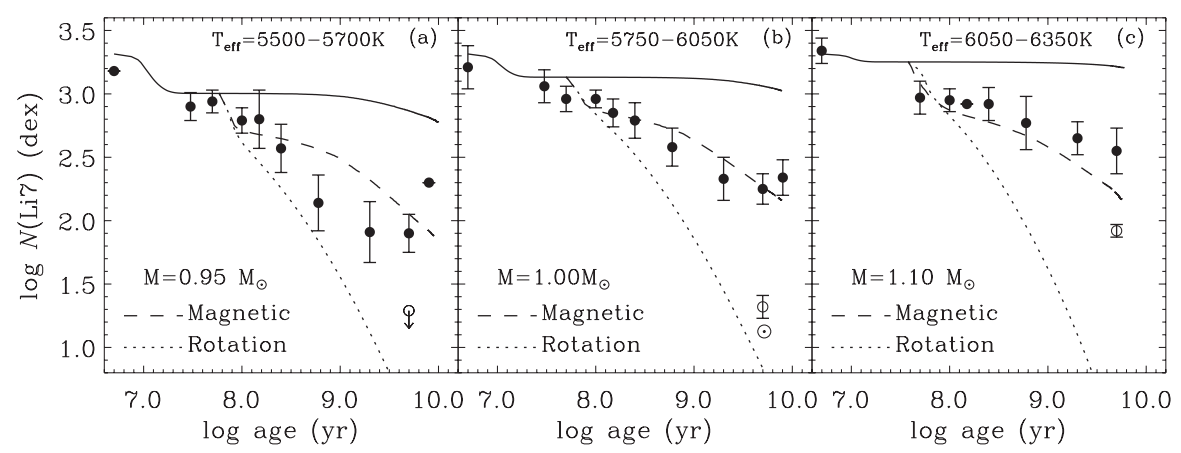

Figure 1. Lithium evolution in solar-type stars in three different $T_{\text {eff }}$ ranges: (a) $T_{\text {eff }}=5500$ $5700 \mathrm{~K}$; (b) $T_{\text {eff }}=5750-6050 \mathrm{~K}$; (c) $T_{\text {eff }}=6050-6350 \mathrm{~K}$. The error bars represent the average $\log N(\mathrm{Li})$ in different clusters as a function of age given by Sestito \& Randich (2005), which is the so-called time scales. In all three figures, the solid lines represent models with diffusion; the dotted lines represent rotating models; models with magnetic fields are showed by dashed lines.

\section{Results}

In this work, we coustructed non-standard models including Tayler-Spruit dynamotype magnetic field to study the effect of magnetic fields on lithium depletion and explain the characters of lithium evolution in solar-type stars. Details of physical mechanism of Tayler-Spruit dynamo and its effects on angular momentum and material can be found in Maeder \& Meynet (2003).

We present our results in Figure 1. The error bars represent the average $\log N(\mathrm{Li})$ in different clusters as a function of age given by Sestito \& Randich (2005), namely, the time scale of lithium evolution. The dashed lines represent lithium depletion reproduced by magnetic models; solid and dotted lines represent lithium evolution of diffusing and rotating models, respectively. For rotating and magnetic models, the initial rotation rate at ZAMS (zero age main sequence) is $50 \Omega_{\odot}$, which is the intermediate value of rotation rates in observed solar-type stars at ZAMS. The relevant studies can be found in Bouvier et al. (1997). As shown in Figure 1, for the late-F and G type stars, lithium burned by models with diffusion is very limited, on the other hand, purely rotating models over deplete lithium at old age. Only the magnetic models can reproduce all the characters of the time scale.

\section{Acknowledgements}

This work is supported by grants 10933002 and 11273007 from the National Natural Science Foundation of China, and the Fundamental Research Funds for the Central Universities.

\section{References}

Chaboyer, B., Demarque, P., \& Pinsonneault, M. H. 1995, ApJ, 411, 865

Bouvier, J., Forestini, M., \& Allain, S. 1997, A\&A, 326, 1023

Maeder, A. \& Meynet, G. 2003, A\&SA, 411, 543

Montalban, J. \& Schatzmann, E. 2000, A\&A A, 354, 943

Deliyannis, C. P. \& Pinsonneault, M. 1997, ApJ, 488, 836

Sestito, P. \& Randich, S. 2005, A\&\&A, 442,615 